\title{
Critical Role of Electrical Resistivity in Magnetoionics
}

\author{
Julius de Rojas $\odot,{ }^{1}$ Joaquín Salguero, ${ }^{2}$ Alberto Quintana $\odot,{ }^{3}$ Aitor Lopeandia,,${ }^{1,4}$ Maciej O. Liedke $\odot,{ }^{5}$ \\ Maik Butterling $\odot,{ }^{5}$ Ahmed G. Attallah $\odot,{ }^{5}$ Eric Hirschman $\odot,{ }^{5}$ Andreas Wagner $\odot,{ }^{5}$ Llibertat Abad, ${ }^{6}$ \\ José L. Costa-Krämer, ${ }^{2, *}$ Jordi Sort $\oplus^{1,7, \dagger}$ and Enric Menéndez ${ }^{1, \grave{\sharp}}$ \\ ${ }^{1}$ Departament de Física, Universitat Autònoma de Barcelona, Cerdanyola del Vallès E-08193, Spain \\ ${ }^{2}$ IMN-Instituto de Micro y Nanotecnología (CNM-CSIC), Isaac Newton 8, PTM, 28760 Tres Cantos, \\ Madrid, Spain \\ ${ }^{3}$ Department of Physics, Georgetown University, Washington, DC 20057, USA \\ ${ }^{4}$ Catalan Institute of Nanoscience and Nanotechnology (ICN2), Campus UAB, Bellaterra, \\ Barcelona, 08193, Spain \\ ${ }^{5}$ Institute of Radiation Physics, Helmholtz-Zentrum Dresden-Rossendorf, Dresden 01328, Germany \\ ${ }^{6}$ Institut de Microelectrònica de Barcelona, IMB-CNM (CSIC), Campus UAB, \\ Cerdanyola del Vallès E-08193, Spain \\ ${ }^{7}$ Institució Catalana de Recerca i Estudis Avançats (ICREA), Pg. Lluís Companys 23, Barcelona E-08010, Spain
}

(Received 8 February 2021; revised 5 July 2021; accepted 2 September 2021; published 23 September 2021)

The utility of electrical resistivity as an indicator of magnetoionic performance in stoichiometrically and structurally similar thin-film systems is demonstrated. A series of highly nanocrystalline cobalt nitride $(\mathrm{Co}-\mathrm{N})$ thin films (85 $\mathrm{nm}$ thick) with a broad range of electrical properties exhibit markedly different magnetoionic behaviors. Semiconducting, near stoichiometric CoN films show the best performance, better than their metallic and insulating counterparts. Resistivity reflects the interplay between atomic bonding, carrier localization, and structural defects, and in turn determines the strength and distribution of applied electric fields inside the actuated films. This fact, generally overlooked, reveals that resistivity can be used to quickly evaluate the potential of a system to exhibit optimal magnetoionic effects, while also opening interesting challenges.

DOI: 10.1103/PhysRevApplied.16.034042

\section{INTRODUCTION}

Modern magnetoelectronic devices, including microand nanoelectromechanical systems, hard disk drives, magnetoresistive random-access memories, etc., are ultimately modulated using electric currents, resulting in significant energy losses through heat dissipation. Voltagecontrolled magnetism, utilizing voltage instead of current to manipulate magnetic properties, might lead to a significant reduction in energy consumption [1,2]. Thus, research in new micro- and nanomaterials, and magnetic actuation methods is critical for developing future miniaturized devices and technologies. Much research has been focused on magnetoionics [3-16], where ions (including $\mathrm{O}, \mathrm{Li}, \mathrm{H}$, $\mathrm{F}$, or $\mathrm{N}$ ) are moved into and out of a target material under an applied electric field, producing large, tunable, nonvolatile changes in magnetic properties, including coercivity, exchange bias field, magnetic easy axis, or anisotropy

\footnotetext{
*jl.costa.kramer@csic.es

†jordi.sort@uab.cat

¥enric.menendez@uab.cat
}

[6,7,10,14,17-23]. Magnetoionic structures are often constituted of ferromagnetic (FM) materials adjacent to an oxide layer, which acts as a solid-state oxygen ion reservoir, such as $\mathrm{GdO}_{x}$ or $\mathrm{HfO}_{x}$. However, such $\mathrm{FM}$ /reservoir bilayer systems often suffer from poor cyclability due to irreversible structural changes undergone by the FM metal in the oxidation (reduction) process, which involves the formation (destruction) of interfacial oxide phases.

An alternative is the use of single-layer target materials whose crystal structure already contains the ions to be transported (such as oxygen or nitrogen) in the asprepared state (e.g., $\mathrm{Co}_{3} \mathrm{O}_{4}$ or $\mathrm{CoN}$ ) $[8,10,24,25]$. Such target materials can undergo fully reversible transformations from a nonferromagnetic (off) to a ferromagnetic (on) state and vice versa while experiencing fewer detrimental structural changes than the previously mentioned magnetoionic approaches, possibly due to $\mathrm{Co}_{3} \mathrm{O}_{4}$ or $\mathrm{CoN}$ structures providing "ready-made" lattice sites into which ions can be driven, leading to increased endurance. In contrast to $\mathrm{Co}_{3} \mathrm{O}_{4}$, where oxygen migration is assisted by the formation of filamentary channels [10], roomtemperature on-off ferromagnetism in CoN films operates 
via frontlike plane-wave ionic motion at lower applied voltages and with enhanced cyclability [24]. Thus, transition metal nitrides compare favorably with their transition metal oxide counterparts for magnetoionic applications.

The magnetoionic effects induced in either single-layer $\left(\mathrm{Co}_{3} \mathrm{O}_{4}, \mathrm{CoN}\right)$ [8] or bilayer $\left(\mathrm{Co} / \mathrm{GdO}_{x}\right)$ [14] systems have been reported to depend on the occurrence of grain boundaries and vacancies (i.e., grain boundaries act as paths for enhanced ionic diffusion [10]), which can be regarded as a tunable component. However, structural defects in general can induce scattering of electrons and therefore changes in electric resistivity [26], which in turn affect the strength of the induced electric field inside the actuated material. The cohesive energy and the strength of interatomic bonds are also key parameters affecting magnetoionic performance, as they are related to the energy required to dissociate cations and anions under the application of electric fields [24]. All these factors (structural defects, grain boundaries, type and strength of atomic bonding) determine electric conductivity [27-34].

Importantly, electric conductivity is also related to the strength and penetration of dc electric fields inside a given layer under an applied external voltage. In metals, the electric field is screened at the surface of the material (within the outermost $0.5 \mathrm{~nm}$ or so) and is zero in its interior. In insulators, the electric field is homogeneous throughout the layer, but its strength is inversely proportional to the layer thickness in a capacitor geometry, while in semiconductors the electric field penetrates several nanometers from the surface towards the interior, progressively decreasing within the so-called Debye length, which is proportional to the dielectric constant and inversely proportional to the carrier density [35-37] (i.e., $\lambda_{D}=\left(\varepsilon k_{B} T / n q^{2}\right)^{1 / 2}$, where $\varepsilon, n, q, k_{B}$, and $T$ denote dielectric constant, charge carrier concentration, electron charge, Boltzmann's constant, and absolute temperature [38]). Thus, inside dc voltageactuated layers the magnitude and distribution of electric field, which is related to the electric resistivity of the target material, is likely to determine the strength of the resulting magnetoionic effects. Despite the expected influence of electric resistivity on magnetoionics, this correlation has been generally overlooked, both in single-layer and in FM/ion-buffer magnetoionic systems.

In this work, a series of cobalt nitride films with variable electrical transport properties are examined. Based on previous reports [39], the Co-N films are grown at relatively high nitrogen partial pressures to ensure nonmagnetic behavior in the as-prepared state (targeting either a face-centered-cubic (fcc) $\mathrm{CoN}$ or a N-rich $\mathrm{Co}_{3} \mathrm{~N}_{1+x}$ phase) [40,41]. Interestingly, previous studies also showed that the crystallite size of fcc $\mathrm{CoN}$ can be significantly decreased with nitrogen partial pressure [39]. Hence, due to enhanced electron scattering at grain boundaries [42], an increase in electric resistivity with increasing nitrogen partial pressure is expected. The differences in magnetoionic behavior of distinct Co-N systems (i.e., between $\mathrm{CoN}$ and $\mathrm{Co}_{3} \mathrm{~N}$ ) are dominated by their dissimilar stoichiometries, crystal structures, and bonding. However, when considering a particular crystal structure and stoichiometry, such as fcc CoN, resistivity is shown to be a reasonably effective indicator that can quickly identify films with functionally superior magnetoionic performance. This underscores the unexplored role that electrical resistivity can play in magnetoionics and ionic motion in solids in general $[43,44]$.

\section{EXPERIMENTAL DETAILS}

The 85 -nm-thick CoN films are grown by reactive sputtering on boron-doped, highly conducting [100] 0.5 -mm-thick silicon wafers, previously coated with $20 \mathrm{~nm}$ of titanium and $60 \mathrm{~nm}$ of copper. The copper is masked during deposition to serve as a working electrode. The expanded $\mathrm{CoN}$ films are grown using a homemade triode sputtering system, with a base pressure in the range of $10^{-8}$ Torr. An ultrahigh vacuum is ensured to minimize oxygen contamination. The target to substrate distances are around $10 \mathrm{~cm}$, and the sputtering rate around $1 \AA \mathrm{s}^{-1}$. $\mathrm{CoN}$ films are grown in an argon-oxygen environment using nitrogen partial pressures of $0 \%, 25 \%, 50 \%, 75 \%$, and $100 \%$, all with a total working pressure of $8 \times 10^{-3}$ Torr.

To characterize defect structure as a function of depth, variable energy positron annihilation lifetime spectroscopy (VEPALS) measurements are conducted at the monoenergetic positron source, an end station of the radiation source ELBE (Electron Linac for beams with high Brilliance and low Emittance) at Helmholtz-Zentrum DresdenRossendorf (Germany) [45], using a $\mathrm{CeBr}_{3}$ detector coupled to a digital lifetime spectrometer with homemade software employing an SP Devices ADQ14DC-2X with 14-bit vertical resolution and 2-GS s${ }^{-1}$ (gigasamples per second) horizontal resolution [46], and a time resolution function down to about $0.205 \mathrm{~ns}$. The resolution function required for spectrum analysis uses two Gaussian functions with distinct intensities depending on the positron implantation energy, $E_{p}$, and appropriate energy shifts. All spectra measured contain at least $10^{7}$ counts.

To investigate the inner parts of the as-grown and biased films, a cross-section lamella is prepared by a focused ion beam and placed onto a $\mathrm{Cu}$ transmission electron microscopy (TEM) grid. To characterize the cross section from structural and compositional viewpoints, high resolution transmission electron microscopy (HRTEM), high-angle annular dark-field scanning transmission electron microscopy (STEM), and electron energy loss spectroscopy (EELS) are performed on a TECNAI F20 HRTEM/STEM microscope operated at $200 \mathrm{kV}$. Further structural characterization is carried out using $\theta / 2 \theta$ 
x-ray diffraction on a Materials Research Diffractometer from the Malvern PANalytical company, equipped with a PIXcel ${ }^{1 \mathrm{D}}$ detector, using $\mathrm{Cu} K \alpha$ radiation. XRD patterns are analyzed using Rietveld refinement to obtain lattice cell parameters and crystallite size (average size of coherently diffracting sample domains) [47].

Magnetoelectric measurements are performed by vibrating sample magnetometry while electrolyte gating the film in a capacitor configuration at room temperature. The samples are mounted in a homemade electrolytic cell containing anhydrous propylene carbonate with sodium cation solvated species (5-25 ppm). The $\mathrm{Na}^{+}$solvated species in the electrolyte are present to react with any trace amounts of water in the propylene carbonate [48]. The magnetic properties of the samples are measured in plane while applying different voltages. This is done using a Micro Sense (LOT - Quantum Design) magnetometer, with a maximum field of $2 \mathrm{~T}$. Voltages are applied using an Agilent B2902A power supply between the sample working electrode and the counter electrode, as demonstrated in previous works $[10,48,49]$. The magnetic signal is normalized to the volume sample exposed to the electrolyte during the gating process. All measured hysteresis loops are background corrected, carried out at high fields (always above the saturation field), to eliminate linear contributions (paramagnetic or diamagnetic signals).

The distribution of the electric field inside the actuated $\mathrm{CoN}$ layers is simulated as a function of electric resistivity using COMSOL software. Note that the electric double layer is modeled as an ultrathin $(0.5 \mathrm{~nm})$ insulating film [50] with relative dielectric constant $\varepsilon_{r}=1$. This value is much smaller than the permittivity of propylene carbonate in the absence of an electric field and is in line with the expected $\varepsilon_{r}$ values under the application of high electric fields generated in the electric double layer [51,52], which could easily exceed $50 \mathrm{Vnm}^{-1}$. The dielectric constant of $\mathrm{CoN}$ was chosen to be around $\varepsilon / \varepsilon_{r}=10$, in agreement with previous reports on several transition metal nitrides [53,54]. To perform resistivity measurements, cobalt nitride films are deposited onto highly resistive $\mathrm{SiO}_{2} / \mathrm{Si}$ substrates. Resistivity values are acquired from 30 to $300 \mathrm{~K}$, all using the van der Pauw configuration.

\section{RESULTS AND DISCUSSION}

To investigate the role of electrical transport properties on magnetoionic performance, cobalt nitride films grown using a range of nitrogen partial pressures $(0 \%-100 \%)$ are evaluated. This variation in partial pressure leads to differing degrees of nanocrystallinity, resulting in a range of semiconducting behavior. Samples are denoted as CoNVL (very low resistivity), CoN-L (low resistivity), CoN$\mathrm{M}$ (medium resistivity), and $\mathrm{CoN}-\mathrm{H}$ (high resistivity), in order of increasing resistivity (Table I).

By comparing $\theta / 2 \theta$ XRD patterns of the as-prepared cobalt nitride films in Figs. 1(a)-1(c), it can be seen that, besides the $\left(\begin{array}{lll}1 & 1 & 1\end{array}\right) \mathrm{Cu}$ peak arising from the buffer layer, the CoN-L, CoN-M, and CoN-H films all exhibit a single broad, low intensity peak that is consistent with the $\left(\begin{array}{lll}1 & 1 & 1\end{array}\right)$ diffraction peak of an expanded $F m \overline{3} m$ cubic CoN phase [PDF (Powder Diffraction File) 00-016-0116], evidencing a polycrystalline structure with strong texture [Fig. 1(b)]. CoN-VL does not present a cubic CoN peak, but rather a broad peak close to the $\left(\begin{array}{lll}1 & 0 & 1\end{array}\right)$ diffraction peak of $\mathrm{Co}_{3} \mathrm{~N}$, suggesting the formation of a highly nanocrystalline, nitrogen-rich $\mathrm{Co}_{3} \mathrm{~N}_{1+x}$ paramagnetic phase [Fig. 1(c)] [39,40]. The XRD pattern of a metallic Co sample is also shown for comparison. Table I lists the nitrogen content by atomic percent for each sample, estimated by energy-dispersive x-ray analysis, and the lattice cell parameters and crystallite or grain sizes, determined by Rietveld refinement of the XRD patterns, of all films. The near-stoichiometric $\mathrm{CoN}$ films (CoN-L, $\mathrm{CoN}-\mathrm{M}$, and $\mathrm{CoN}-\mathrm{H}$ ) exhibit expanded lattice parameters of approximately $3 \%$ and crystallite sizes ranging from 6 to $13 \mathrm{~nm}$, while the $\mathrm{Co}_{3} \mathrm{~N}_{1+x}$ film (CoN-VL) exhibits crystallite sizes around $9 \mathrm{~nm}$.

Since the ability to hold an electric field and the electric field's strength are crucial components in enabling magnetoionics, a thorough electric transport characterization is carried out [Fig. 1(d)]. Measurements are also conducted on a purely metallic cobalt film as reference. Decreasing the crystallite size $\langle D\rangle$ clearly increases the resistivity in the near-stoichiometric $\mathrm{CoN}$ films, from approximately $411 \mu \Omega \mathrm{cm}$ in CoN-L to approximately $1200 \mu \Omega \mathrm{cm}$ in $\mathrm{CoN}-\mathrm{H}$, at room temperature (Table I). The crystallite size

TABLE I. Cobalt nitride samples, nitrogen partial pressure during sputtering, nitrogen concentration, relative lattice expansion, crystallite size, resistivity at room temperature, and relative electric behavior. ${ }^{\text {a }}$

\begin{tabular}{lcccccc}
\hline \hline Co-N & $P_{N 2}(\%)$ & N Concentration $(\%)$ & $\Delta a / a_{0}(\%)$ & $\langle D\rangle(\mathrm{nm})$ & $\rho(\mu \Omega \mathrm{cm})$ & Electric behavior $(d \rho / d T)$ \\
\hline CoN-VL & 25 & 35 & N/A & 9 & 156 & Metallic \\
CoN-L & 50 & 50 & +3.50 & 13 & 411 & Metallic/Semiconducting \\
CoN-M & 75 & 48 & +3.06 & 8 & 1104 & Semiconducting \\
CoN-H & 100 & 47 & +3.02 & 6 & 1126 & Semiconducting/Insulating \\
\hline \hline
\end{tabular}

${ }^{a}$ Lattice parameters and crystallite sizes are obtained from Rietveld refinement of the XRD patterns [47]. Theoretical lattice parameter of $\mathrm{Fm} \overline{3} \mathrm{~m}$ phase $\mathrm{CoN}$ is taken to be $a_{0}=4.28 \AA$ (PDF 00-016-0116). Resistivity of as-prepared, pure cobalt was measured to be $11 \mu \Omega \mathrm{cm}$ at room temperature. 
(a)

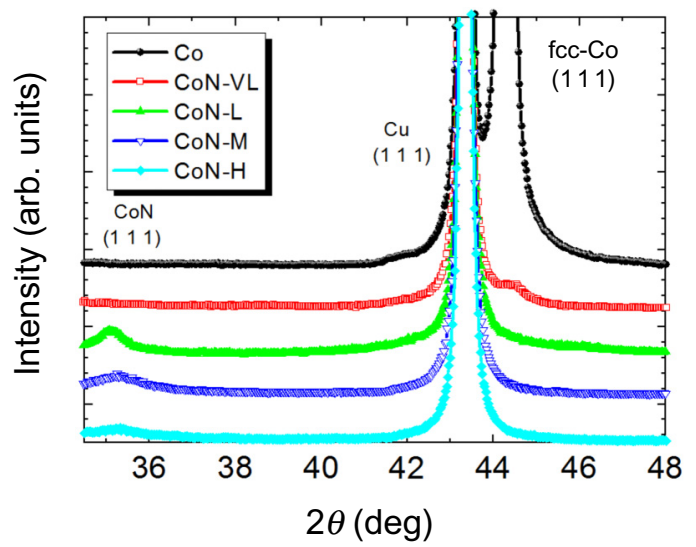

(c)

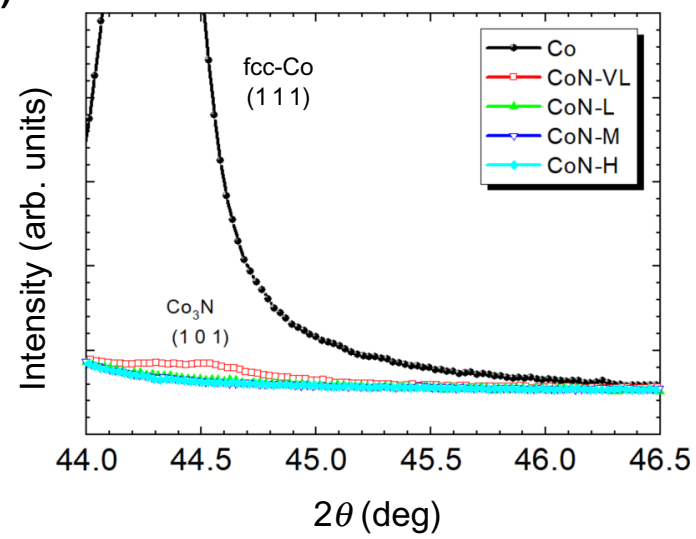

(e)

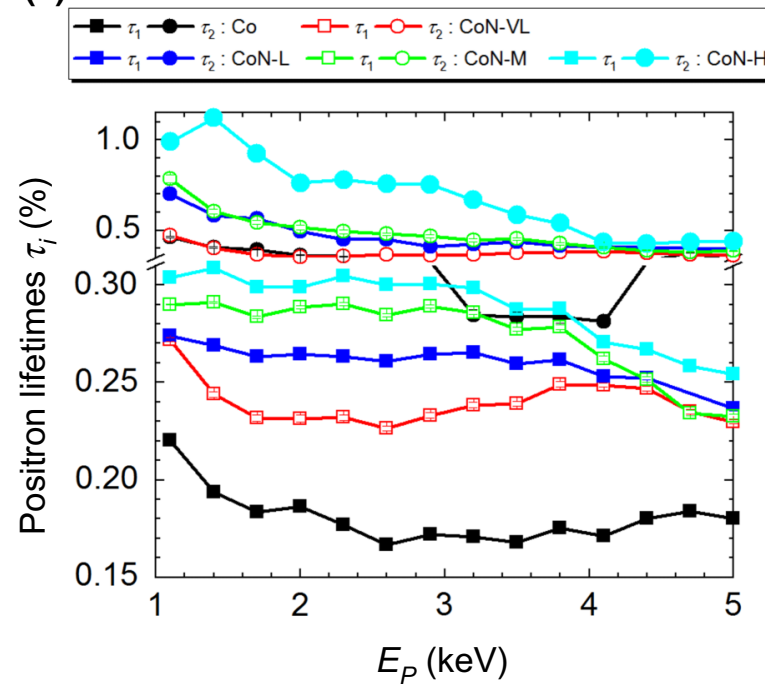

(b)

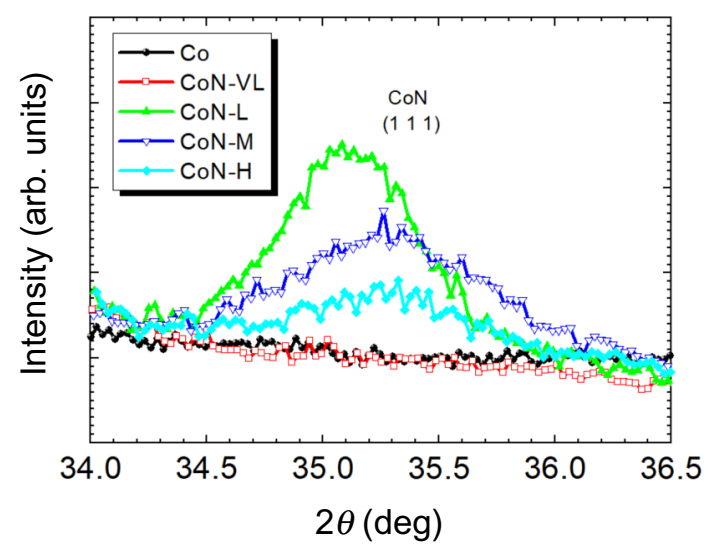

(d)

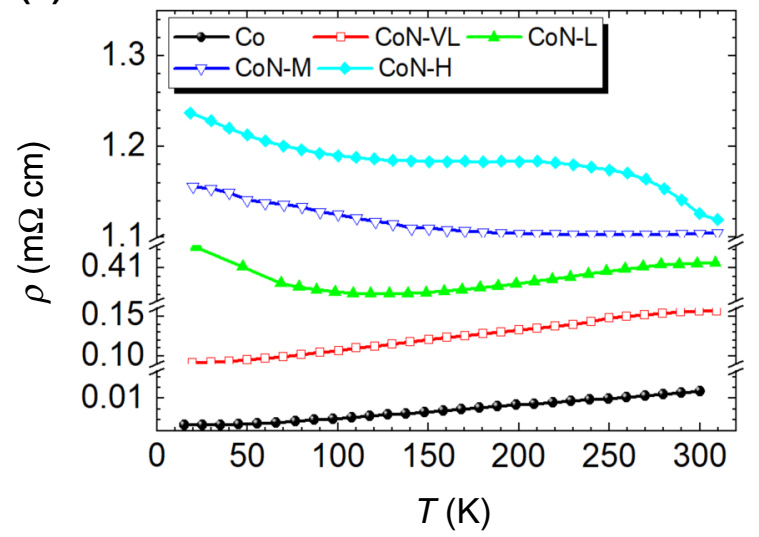

(f)

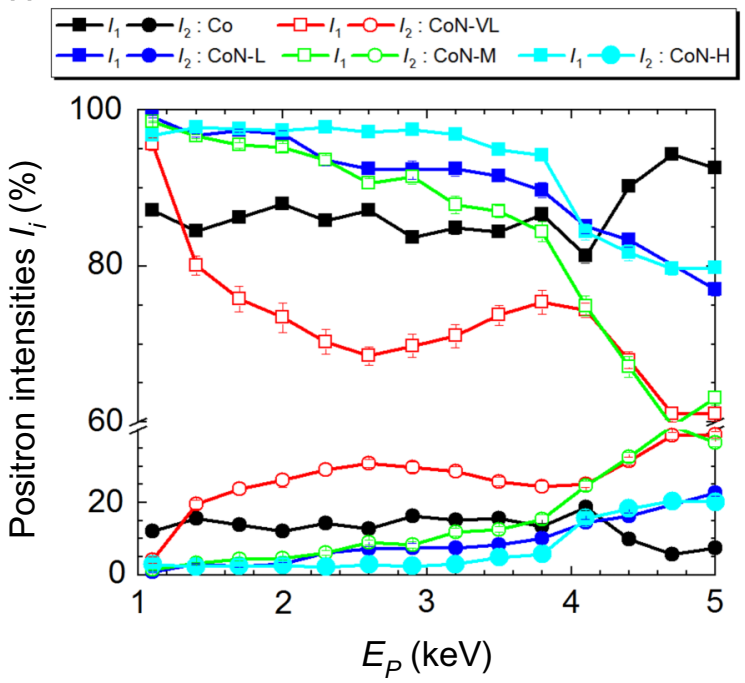

FIG. 1. (a) $\theta / 2 \theta$ XRD diffraction patterns of the as-prepared cobalt nitride films. (b) and (c) Enlarged $\theta / 2 \theta$ XRD diffraction patterns of near-stoichiometric CoN films (card number PDF 00-016-0116) and $\mathrm{Co}_{3} \mathrm{~N}$ [40], respectively. (d) Resistivity $\rho$ measured as a function of temperature from 20 to $300 \mathrm{~K}$, for all cobalt nitride as-prepared samples. (e) and (f) positron lifetime components $\tau_{i=1-2}$ and relative intensities $I_{i=1-2}$, respectively, as a function of positron implantation energy $E_{P}$ for all as-prepared cobalt nitride samples. 
$\langle D\rangle$ of CoN-VL is smaller than for CoN-L $(9 \mathrm{~nm})$, but it should be noted that the phases are distinct $\left(\mathrm{Co}_{3} \mathrm{~N}_{1+x}\right.$ versus $\mathrm{CoN}$ ); as such, the crystallite size plays a secondary role when comparing across phases. The pure cobalt sample shows a monotonic increase of resistivity throughout the temperature range $(d \rho / d T>0$, where $\rho$ and $T$ are resistivity and temperature, respectively), consistent with metallic behavior (for plots of individual curves see Fig. S1 in Supplemental Material [55]). In CoN-VL, the resistivity is observed to monotonically increase as well (metalliclike), albeit with a higher overall resistivity (approximately $156 \mu \Omega \mathrm{cm}$ ) than pure cobalt throughout the temperature range due to the presence of nitrogen $[56,57]$. In CoN-L, a parabolic shape is observed with semiconducting behavior $(d \rho / d T<0)$ appearing below $100 \mathrm{~K}$, and metallic transport $(d \rho / d T>0)$ above $100 \mathrm{~K}$. CoN-M shows an overall semiconducting behavior, while CoN-H behaves similarly, but exhibits a larger resistivity. Thus, the nitrided samples can be considered to lie on a metallic-semiconducting spectrum, with CoN-VL the most conductive and CoN-H the most resistive (Table I). This suggests that the interplay between nitrogen concentration and microstructure plays an important role in the dielectric.

To further understand the microstructure of each film, VEPALS experiments are conducted [45,58-62]. VEPALS data for each as-prepared cobalt nitride film is presented. Only contributions from $\tau_{1}$, corresponding to localized vacancies and vacancy clusters, and $\tau_{2}$, corresponding to a mixture of signals from surface states and grain boundaries are observed [Figs. 1(e) and 1(f)] [10]. It should be noted that, in the cases of smaller grain sizes (as seen in films sputtered with high nitrogen partial pressure), $\tau_{1}$ could also represent grain boundaries, as the positrons annihilate in the largest open volumes, corresponding here to grain boundaries. For $E_{P}>3.5 \mathrm{keV}$ a change is observed for all parameters of the cobalt nitride films in Figs. 1(e) and 1(f). This feature is likely to be a fingerprint of a different microstructure defect (fewer vacancies) appearing deeper in the film, closer to the film/buffer $(\mathrm{Cu} / \mathrm{Ti})$ interface. As $E_{P}$ increases, the signal from the substrate emerges, and each line asymptotically approaches a plateau. In general, positron lifetimes $\tau_{1}$ and $\tau_{2}$ are observed to increase with resistivity, indicating a substantial rise in the defect size (see Fig. S2 in Supplemental Material [55] for details). The intensity of $\tau_{1}, I_{1}$, is lowest for CoN-VL, then increases for $\mathrm{CoN}-\mathrm{L}$ and $\mathrm{CoN}-\mathrm{M}$, reaching a maximum for $\mathrm{CoN}-\mathrm{H}$, with $I_{2}$ contributing only residual amounts at high nitrogen concentration and indicating an overall increase in effective defect size (see Fig. S3 in Supplemental Material [55] for the average positron lifetime). Thus, as expected, structural defects have a detrimental effect on electric conductivity, in agreement with other systems, such as metals, in which an increase in the number of vacancies leads to greater electronic scattering and so is correlated with larger resistivities [63].
Figure 2(a) shows the capacitor (condenserlike) structure used to generate the electric field in all reported measurements. Propylene carbonate (with dissolved $\mathrm{Na}^{+}$) is used as a nonaqueous, aprotic, polar electrolyte that allows for the application of a uniform voltage by creating an electric double layer at the film surface [10]. Voltage is applied while in-plane hysteresis loops are recorded by vibrating sample magnetometry $[18,49,64-67]$. In this configuration, a $\mathrm{Cu} / \mathrm{Ti}$ buffer layer acts as a working electrode and a $\mathrm{Pt}$ wire acts a counter electrode, so an out-of-plane electric field is initially generated $[8,49]$. Asprepared CoN-VL, CoN-L, CoN-M, and CoN-H samples show residual FM signals between 1 and $8 \mathrm{emu} \mathrm{cm}^{-3}$ [see Fig. S4(a) in Supplemental Material [55] ], with a small initial magnetization present in some samples possibly due to residual off-stoichiometric regions in the film, FM impurities in the substrate, or $\mathrm{CoN}$ reduction at the $\mathrm{CoN} / \mathrm{Cu}$ interface.

The off-stoichiometry may extend across the film, consistent with the difference in defect structure between the top and bottom of the as-prepared films observed in the VEPALS data [Fig. 1(e)]. In any case, the measured saturation magnetization, $M_{S}$, is very low and it corresponds to approximately $0.56 \%$ of metallic Co by volume.

To study their magnetoionic behavior, the cobalt nitride films are biased under $-50 \mathrm{~V}$ during a sequence of $15 \mathrm{con}$ secutive magnetic hysteresis loops, lasting $25 \mathrm{~min}$ each. During the first loop, the samples show hysteric behavior and a clear increase in magnetization $(M)$ as time evolves, indicating the clear emergence of a FM phase. The first loop measured during each biasing protocol is shown for each film in Fig. 2(b). The magnetization of CoN-VL (i.e., $\mathrm{Co}_{3} \mathrm{~N}_{1+x}$ ) increases under bias, reaching maximal magnetization $\left(90 \mathrm{emu} \mathrm{cm}^{-3}\right)$ under $-50 \mathrm{~V}$ gating after one loop (25 min). As reported earlier [24], CoN-L (denoted in the cited work as "CoN") shows a tremendous increase in magnetic signal while sweeping through the first quadrant (approximately $6 \mathrm{~min}$ ) of the hysteresis loop under bias (red loops), and then nearly doubling this value by the end of the first hysteresis loop, to $630 \mathrm{emu} \mathrm{cm}^{-3}$, a factor of nearly seven when compared with CoN-VL during the same time frame $(25 \mathrm{~min})$. The $\mathrm{CoN}-\mathrm{M}$ and $\mathrm{CoN}-\mathrm{H}$ samples reach values nearly one-half and one-third as large as CoN-L, respectively, albeit with a lower rate of increase, as clearly expected from the much milder increase in $M$ as the applied magnetic field, $H$, is swept through the first quadrant [for several loops plotted in sequence, see Figs. S4(b)-S4(e) in Supplemental Material [55] ].

To evaluate the rate of increase of the magnetic signal, the saturation magnetization, $M_{S}$, is plotted as a function of time for all films [Fig. 2(c)]; see previous works for further details on quantification $[8,24]$. Both CoN-VL and CoN-L show a rapid increase (CoN-L especially) and reach magnetic saturation during the first loop, although all samples show a clear increase in $M_{S}$ with time. CoN-VL reaches 
(a)
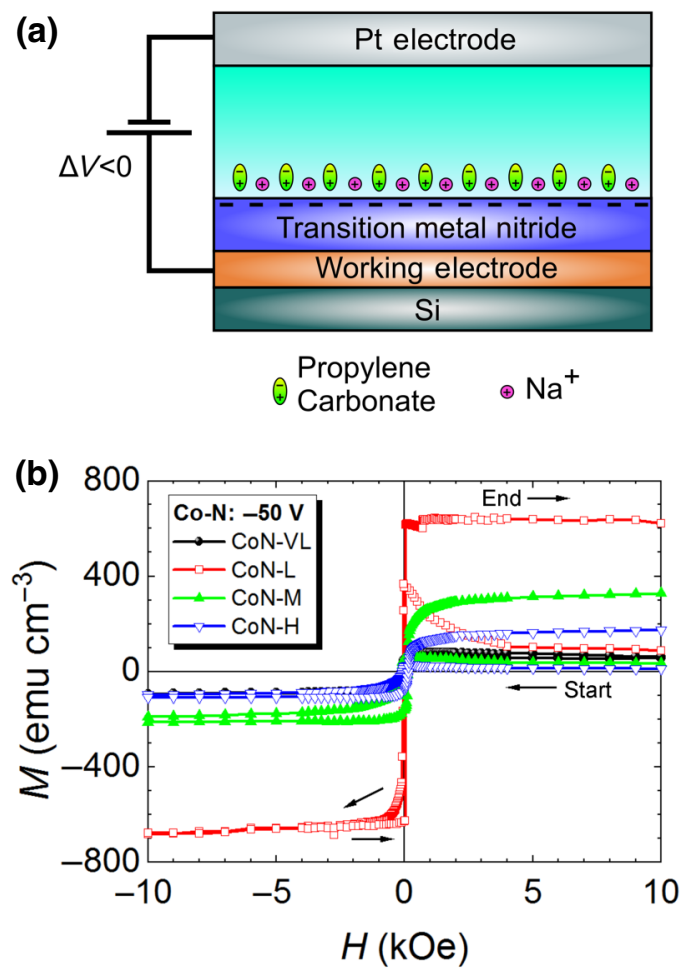

(c)

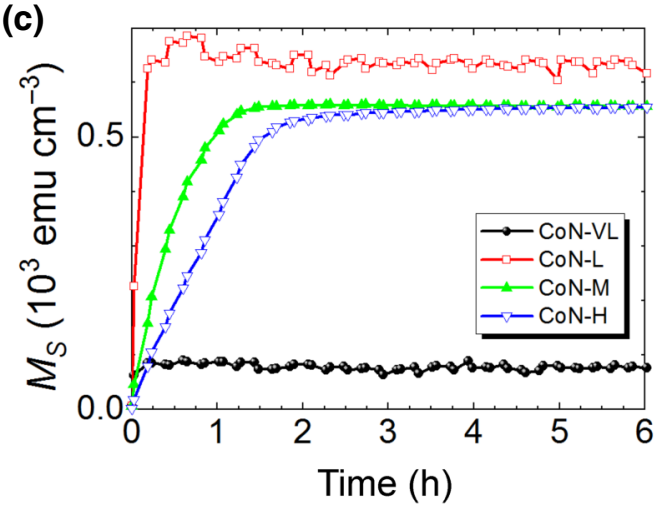

FIG. 2. (a) Schematic of the condenserlike structure used in each film (electrochemical capacitor configuration). (b) First hysteresis loops under $-50 \mathrm{~V}$ bias for cobalt nitride films. (c) Saturation magnetization $\left(M_{S}\right)$ measured as a function of time for cobalt nitride films.

a maximal value of $M_{S}$ near $90 \mathrm{emu} \mathrm{cm}^{-3}$, while all other samples reach a maximal $M_{S}$ greater than $550 \mathrm{emu} \mathrm{cm}^{-3}$ (Table II). Both CoN-M and CoN-H reach much higher $M_{S}$ values than CoN-VL, although less than CoN-L. The smaller maximal $M_{S}$ value of the $\mathrm{Co}_{3} \mathrm{~N}_{1+x}(\mathrm{CoN}-\mathrm{VL})$ film under biasing also points towards an interesting result; despite its proximity to a $\mathrm{FM} \mathrm{Co}_{3+x} \mathrm{~N}$ phase possessing a sizable $M_{S}$ [68], ionic motion terminates early in the biasing process, limiting the observed increase in magnetization. To elucidate the timescales of increasing ferromagnetism from the as-prepared state, the slope of the magnetization increases during the first $12 \mathrm{~min}$ is fitted using a linear regression. The rates obtained during this period are $1012 \mathrm{emu} \mathrm{cm}^{-3} \mathrm{~h}^{-1}$ for CoN-VL, $2602 \mathrm{emu} \mathrm{cm}^{-3} \mathrm{~h}^{-1}$ for CoN-L, $723 \mathrm{emu} \mathrm{cm}^{-3} \mathrm{~h}^{-1}$ for CoN-M, and $427 \mathrm{emu} \mathrm{cm}^{-3} \mathrm{~h}^{-1}$ for CoN-H, showing that, under equivalent voltage, CoN-L generates a FM state at a significantly higher rate. For further examination of the evolution of the magnetic and structural properties of the samples under biasing similar to those done in previous works $[8,24]$ (i.e., coercivity, squareness, slope of the hysteresis loops, XRD), see Figs. S5 and S6 in Supplemental Material [55].

There is a clear variation in magnetoionic performance between $\mathrm{Co}_{3} \mathrm{~N}_{1+x}$ and $\mathrm{CoN}$ phases, which is reflected in their overall magnetization and magnetic properties under biasing, as can be expected with films of differing stoichiometry and structure. However, even when considering a family of structurally and stoichiometrically similar films, distinct magnetoionic effects are clearly observed. Contrary to what has been reported in previous works, the increase in grain boundaries and nanocrystallinity (directly related to resistivity) of the CoN-M and $\mathrm{CoN}-\mathrm{H}$ samples, on their own, do not correlate with increased magnetoionic effects [10]; in fact, the film with the highest magnetoionic rate $(\mathrm{CoN}-\mathrm{L})$ does not possess the largest number of defects or grain boundaries (lower resistivity). This, in conjunction with the limited increase in $M_{S}$ in CoNVL, suggests a complementary effect that counterbalances the effect of increasing ionic conduction pathways and ionic mobility. In particular, the electric field driving ionic motion in the film may be suppressed near the surface due to the Debye screening effect, which is related to the charge carrier density and dielectric properties of the film. To estimate the field profile inside the film, COMSOL simulations are performed for an $85-\mathrm{nm}$ semiconducting film with a range of charge carrier densities $\left(n=10^{15}-10^{21}\right)$ at the moment $(t=0)$ a bias of $-50 \mathrm{~V}$ is applied via an electric double layer, before ion motion begins (Fig. 3). The Debye length is examined for a relative permittivity value of $\varepsilon_{r}=10$, representing a typical value for transition metal nitrides [53,54]. The calculated electric fields are normalized to the field of a highly resistive film $\left(n=10^{5}\right.$ carriers) taken to be insulating. The carrier density is found to modify the penetration depth of the field, ranging from $<1 \mathrm{~nm}$ up to $>10 \mathrm{~nm}$. In the metalliclike CoN-VL film (with a larger number of charge carriers), the electric field is very high at the interface with the propylene carbonate, but goes to zero very rapidly within the first 1-2 nm of the surface. In this case, the applied voltage is able to induce $\mathrm{N}$ ion motion only at the interface with the electrolyte, leaving most of the layer unaffected, and thus rendering very small magnetoionic effects beyond a quickly achieved maximum [in agreement with the results shown in Fig. 2(b)]. In contrast, in the most insulating film $(\mathrm{CoN}-\mathrm{H})$ the applied voltage produces a field that extends deep into the film, but with relatively low strength. In the 
TABLE II. Magnetoionic rates under $-50 \mathrm{~V}$ bias during the first $12 \mathrm{~min}$, saturation magnetization, loop squareness, normalized slope at the coercive field, and coercive field after $6 \mathrm{~h}$. All values are measured under $-50 \mathrm{~V}$ bias.

\begin{tabular}{lccccc}
\hline \hline Co-N films & $d M_{S} / d t\left(\mathrm{emu} \mathrm{cm}^{-3} \mathrm{~h}^{-1}\right)$ & $M_{S}\left(\mathrm{emu} \mathrm{cm}^{-3}\right)$ & $M_{R} / M_{S}(\%)$ & $M_{S}^{-1} d M / d H @ H_{C}\left(\mathrm{kOe}^{-1}\right)$ & $H_{C}(\mathrm{Oe})$ \\
\hline CoN-VL & 1012 & 90 & 84 & 21 & 65 \\
CoN-L & 2602 & 637 & 96 & 97 & 17 \\
CoN-M & 723 & 558 & 83 & 18 & 153 \\
CoN-H & 427 & 556 & 80 & 16 & 167 \\
\hline \hline
\end{tabular}

intermediate case (semiconducting CoN layers with intermediate resistivity values), a substantially larger electric field is obtained near the interface, propagating $>10 \mathrm{~nm}$ inwards, affecting a larger volume of the magnetoionic layer than CoN-VL. Additionally, the defect gradient along the depth, as observed by PALS, together with the smaller, isotropic grains (as compared with the columnar grains in $\left.\mathrm{Co}_{3} \mathrm{O}_{4}[8,10]\right)$, may promote the planar ionic front seen in $\mathrm{CoN}$ [24]. It should be noted that the physical picture presented in Fig. 3 shows the penetrating field $E$ after the charge carriers have redistributed along the surface under an applied voltage, which alone does not capture all the observed behavior.

This model only provides an approximation of the initial steady-state electric field, however; the dynamics of ionic motion (i.e., how $\mathrm{N}$ ions are transported and charge

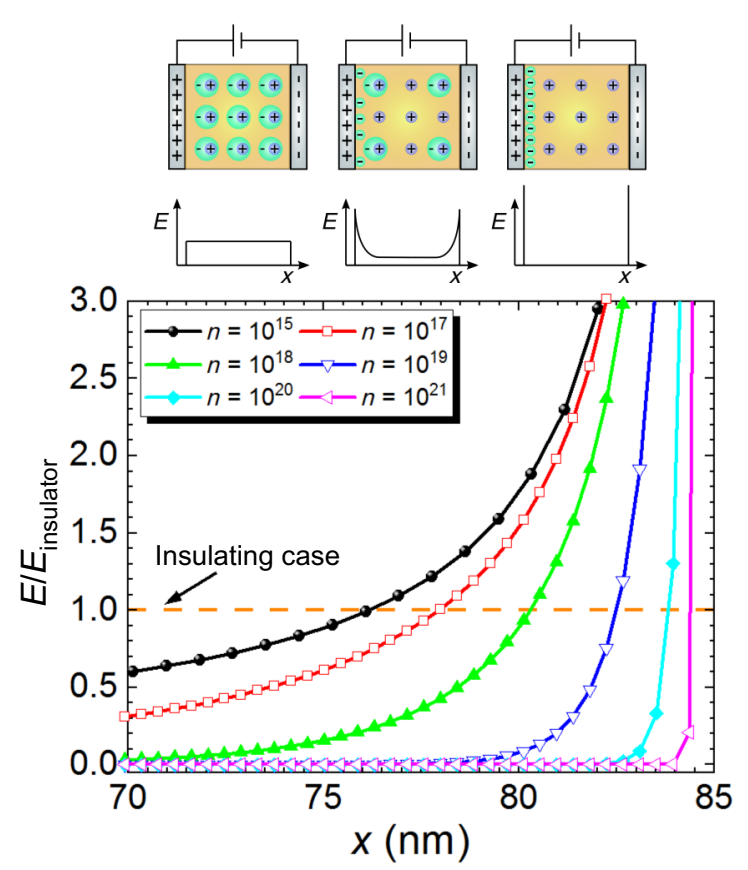

FIG. 3. Debye screening lengths calculated for charge carrier densities ranging from $n=10^{15}-10^{21}$ (least to most insulating cases), calculated for a typical relative permittivity for transition metal nitrides of $\varepsilon_{r}=10$ and normalized to the resistive case. Simple schematics above show representative expected field extensions in the ideal insulating (left), semiconducting (center), and metallic cases (right). carriers in the denitrided area redistributed) must play a key role in magnetoionic performance. Here, we sketch a plausible description for ionic motion under constant bias. For intermediate resistivity values, the electric field is strong enough to eventually produce nitrogen ionization and ion migration into the electrolyte, thereby leaving positively charged nitrogen vacancies behind. As ionization proceeds, nitrogen vacancies flow inwards towards the negatively charged working electrode and an equivalent, oppositely charged nitrogen ionic current moves towards the electric double layer. Nitrogen ions are driven outside the material layer-by-layer (onionlike) via plane-wave-like migration, leaving behind an electrochemical gradient that may vary at grains and grain boundaries, impacting the penetrating field distribution and local ion redistribution. Note that as ion migration proceeds, the resistivity profile of the CoN will change, as observed in other systems during ionic movement [53]. During ion migration, semiconducting $\mathrm{CoN}$ becomes more electrically conductive due to the formation of metallic Co clusters and, with the increase in charge carrier density, a decrease in the Debye length. This is a dynamic and coupled effect that depends on electrical resistivity and the screening length; as ionic flow progresses and the penetration depth of the electric field progressively decreases, the field reaches less of the layer interior, leading to a decrease in magnetoionic effects, eventually reaching an equilibrium in the generated magnetization, as observed as a saturation after several hours of biasing [24]. This process continues until the nitrogen is exhausted or the induced metallic cobalt is able to screen the applied electric field. Note that the attained value of $M_{S}$ is close to $700 \mathrm{emu} \mathrm{cm}^{-3}$ [Fig. 2(c)], which roughly corresponds to half of the $1422 \mathrm{emu} \mathrm{cm}^{-3} M_{S}$ of pure Co [69], suggesting that the layer becomes highly nanoporous [24] since, given the absence of $\mathrm{N}$ (as assessed by EELS), no contribution from $\mathrm{Co}_{4} \mathrm{~N}$ or $\mathrm{Co}_{3+x} \mathrm{~N}$ phases to the magnetic properties is expected.

The role of resistivity in the generated electric field must be refined, also taking into account the strength of the CoN atomic bonding [70] to better understand the dissociation of the $\mathrm{CoN}$ molecules in the proximity of the electrolyte. The bond strength increases in more resistive bonds, such as $\mathrm{CoN}-\mathrm{H}$, while more conductive bonds, such as those in CoN-VL, are typically associated with weaker atom-atom bonds, which can potentially facilitate ionic 
motion. In particular, CoN-VL shows a mild increase in $M_{S}$ before quickly flattening, while the more resistive CoN-L shows a large increase in magnetization, much greater than CoN-VL. This difference can be attributed to the different stoichiometries and crystal structures of the two films $\left(\mathrm{Co}_{3} \mathrm{~N}_{1+x}\right.$ and $\left.\mathrm{CoN}\right)$, but not so in the three $\mathrm{CoN}$ films. The even more resistive CoN-M and CoN-H films both show milder increases in $M_{S}$ than the relatively conducting CoN-L. Each of the factors considered (defect density, microstructure, screening length) are reflected in the electrical transport properties of the sample, and this suggests a balance must be reached between the extension of the applied field into the film, the density of ionic conduction pathways, and the bond strength holding ions in place. For a given crystal structure and stoichiometry, these properties can be tuned via defects and microstructure, with resistivity serving as a quick and easily measured indicator of magnetoionic potential. The influence of the detailed grain structure on the field and ionic motion profiles, presumably with grains as more coordinated structures with larger resistivity and grain boundaries as less coordinated and more conductive, is an interesting point that deserves further future investigation.

\section{CONCLUSION}

Electrical resistivity is a key indicator for magnetoionic motion among materials of similar structure and stoichiometry (and can thus be used to identify which film is best suited for magnetoionic applications) because it crucially merges the interplay between transition metal - nitrogen bonding, microstructure, size and type of defects, and the strength of the electric field and its corresponding penetration depth into the target material. $\mathrm{Co}_{3} \mathrm{~N}_{1+x}$ films are found to exhibit a very limited magnetoionic response due to electric-field screening, while the more insulating $\mathrm{CoN}$ films show improved ionic motion, albeit with a range of magnetoionic rates, which can be quickly assessed via electric transport measurements. The best balance is achieved in $\mathrm{CoN}$ films with intermediate resistivity values. This work not only has implications in nitrides, but also applies more generally to other singlelayer semiconductors, and perhaps also provides a powerful indicator in tuned bilayer systems (such as $\mathrm{Co} / \mathrm{GdO}_{x}$ or $\mathrm{Co} / \mathrm{HfO}_{x}$ ) that can be used to optimize magnetoionic effects.

\section{ACKNOWLEDGMENTS}

Financial support by the European Research Council (SPIN-PORICS 2014-Consolidator Grant, Agreement No. 648454, and the MAGIC-SWITCH 2019-Proof of Concept Grant, Agreement No. 875018), the Spanish Government (MAT2017-86357-C3-1-R and PID2020116844RB-C21), the Generalitat de Catalunya (2017SGR-292 and 2018-LLAV-00032) and the European
Regional Development Fund (MAT2017-86357-C3-1-R and 2018-LLAV-00032) is acknowledged. This work was partially supported by the Impulse-und Net-working fund of the Helmholtz Association (FKZ VH-VI-442 Memriox), and the Helmholtz Energy Materials Characterization Platform (03ET7015). The PALS measurements were carried out at ELBE at the Helmholtz-Zentrum DresdenRossendorf e. V., a member of the Helmholtz Association. L.A. thanks MINECO for a Ramón y Cajal Contract (RYC-2013-12640). J.S. thanks the Spanish Fábrica Nacional de Moneda y Timbre for fruitful discussions. E.M. acknowledges support as a Serra Húnter Fellow. We acknowledge service from MiNa Laboratory at IMNCSIC.

[1] C. Song, B. Cui, F. Li, X. Zhou, and F. Pan, Recent progress in voltage control of magnetism: Materials, mechanisms, and performance, Prog. Mater. Sci. 87, 33 (2017).

[2] J. M. Hu and C. W. Nan, Opportunities and challenges for magnetoelectric devices, APL Mater. 7, 80905 (2019).

[3] S. Vasala, A. Jakob, K. Wissel, A. I. Waidha, L. Alff, and O. Clemens, Reversible tuning of magnetization in a ferromagnetic ruddlesden-popper-type manganite by electrochemical fluoride-ion intercalation, Adv. Electron. Mater. 6, 1900974 (2020).

[4] A. J. Tan, M. Huang, S. Sheffels, F. Büttner, S. Kim, A. H. Hunt, I. Waluyo, H. L. Tuller, and G. S. D. Beach, Hydration of gadolinium oxide ( $\mathrm{Gd} \mathrm{Ox}$ ) and its effect on voltage-induced $\mathrm{Co}$ oxidation in a $\mathrm{Pt} / \mathrm{Co} / \mathrm{Gd} \mathrm{Ox} / \mathrm{Au}$ heterostructure, Phys. Rev. Mater. 3, 064408 (2019).

[5] S. Dasgupta, B. Das, M. Knapp, R. A. Brand, H. Ehrenberg, R. Kruk, and H. Hahn, Intercalation-driven reversible control of magnetism in bulk ferromagnets, Adv. Mater. 26, 4639 (2014).

[6] U. Bauer, L. Yao, A. J. Tan, P. Agrawal, S. Emori, H. L. Tuller, S. Van Dijken, and G. S. D. Beach, Magnetoionic control of interfacial magnetism, Nat. Mater. 14, 174 (2015).

[7] L. Baldrati, A. J. Tan, M. Mann, R. Bertacco, and G. S. D. Beach, Magneto-ionic effect in CoFeB thin films with in-plane and perpendicular-to-plane magnetic anisotropy, Appl. Phys. Lett. 110, 012404 (2017).

[8] J. de Rojas, A. Quintana, A. Lopeandía, J. Salguero, J. L. Costa-Krämer, L. Abad, M. O. Liedke, M. Butterling, A. Wagner, L. Henderick, J. Dendooven, C. Detavernier, J. Sort, and E. Menéndez, Boosting room-temperature magneto-ionics in a non-magnetic oxide semiconductor, Adv. Funct. Mater. 30, 2003704 (2020).

[9] A. J. Tan, M. Huang, C. O. Avci, F. Büttner, M. Mann, W. Hu, C. Mazzoli, S. Wilkins, H. L. Tuller, and G. S. D. Beach, Magneto-ionic control of magnetism using a solid-state proton pump, Nat. Mater. 18, 35 (2019).

[10] A. Quintana, E. Menéndez, M. O. Liedke, M. Butterling, A. Wagner, V. Sireus, P. Torruella, S. Estradé, F. Peiró, J. Dendooven, C. Detavernier, P. D. Murray, D. A. Gilbert, K. Liu, E. Pellicer, J. Nogues, and J. Sort, Voltage-controlled ON-OFF ferromagnetism at room temperature in a single metal oxide film, ACS Nano 12, 10291 (2018). 
[11] A. Molinari, H. Hahn, and R. Kruk, Voltage-control of magnetism in all-solid-state and solid/liquid magnetoelectric composites, Adv. Mater. 31, 1806662 (2019).

[12] M. Gößler, M. Albu, G. Klinser, E. M. Steyskal, H. Krenn, and R. Würschum, Magneto-ionic switching of superparamagnetism, Small 15, 1904523 (2019).

[13] D. A. Gilbert, J. Olamit, R. K. Dumas, B. J. Kirby, A. J. Grutter, B. B. Maranville, E. Arenholz, J. A. Borchers, and K. Liu, Controllable positive exchange bias via redoxdriven oxygen migration, Nat. Commun. 7, 11050 (2016).

[14] D. A. Gilbert, A. J. Grutter, E. Arenholz, K. Liu, B. J. Kirby, J. A. Borchers, and B. B. Maranville, Structural and magnetic depth profiles of magneto-ionic heterostructures beyond the interface limit, Nat. Commun. 7, 12264 (2016).

[15] K. Duschek, M. Uhlemann, H. Schlörb, K. Nielsch, and K. Leistner, Electrochemical and in situ magnetic study of iron/iron oxide films oxidized and reduced in $\mathrm{KOH}$ solution for magneto-ionic switching, Electrochem. Commun. 72, 153 (2016).

[16] S. Dasgupta, B. Das, Q. Li, D. Wang, T. T. Baby, S. Indris, M. Knapp, H. Ehrenberg, K. Fink, R. Kruk, and H. Hahn, Toward on-and-off magnetism: Reversible electrochemistry to control magnetic phase transitions in spinel ferrites, Adv. Funct. Mater. 26, 7507 (2016).

[17] F. Ibrahim, A. Hallal, B. Dieny, and M. Chshiev, Establishing characteristic behavior of voltage control of magnetic anisotropy by ionic migration, Phys. Rev. B 98, 214441 (2018).

[18] C. Navarro-Senent, J. Fornell, E. Isarain-Chávez, A. Quintana, E. Menéndez, M. Foerster, L. Aballe, E. Weschke, J. Nogués, E. Pellicer, and J. Sort, Large magnetoelectric effects in electrodeposited nanoporous microdisks driven by effective surface charging and magneto-ionics, ACS Appl. Mater. Interfaces 10, 44897 (2018).

[19] S. A. Wolf, D. D. Awschalom, R. A. Buhrman, J. M. Daughton, S. von Molnár, M. L. Roukes, A. Y. Chtchelkanova, and D. M. Treger, Spintronics: A spin-based electronics vision for the future, Science 294, 1488 (2001).

[20] E. H. Khan, M. H. Weber, and M. D. McCluskey, Formation of Isolated $\mathrm{Zn}$ Vacancies in $\mathrm{ZnO}$ Single Crystals by Absorption of Ultraviolet Radiation: A Combined Study Using Positron Annihilation, Photoluminescence, and Mass Spectroscopy, Phys. Rev. Lett. 111, 017401 (2013).

[21] W. Eerenstein, N. D. Mathur, and J. F. Scott, Multiferroic and magnetoelectric materials, Nature 442, 759 (2006).

[22] K. Duschek, A. Petr, J. Zehner, K. Nielsch, and K. Leistner, All-electrochemical voltage-control of magnetization in metal oxide/metal nanoislands, J. Mater. Chem. C 6, 8411 (2018).

[23] C. Bi, Y. Liu, T. Newhouse-Illige, M. Xu, M. Rosales, J. W. Freeland, O. Mryasov, S. Zhang, S. G. E. Te Velthuis, and W. G. Wang, Reversible Control of Co Magnetism by Voltage-Induced Oxidation, Phys. Rev. Lett. 113, 267202 (2014).

[24] J. de Rojas, A. Quintana, A. Lopeandía, J. Salguero, B. Muñiz, F. Ibrahim, M. Chshiev, A. Nicolenco, M. O. Liedke, M. Butterling, A. Wagner, V. Sireus, L. Abad, C. J. Jensen, K. Liu, J. Nogués, J. L. Costa-Krämer, E. Menéndez, and J. Sort, Voltage-driven motion of nitrogen ions: A new paradigm for magneto-ionics, Nat. Commun. 11, 5871 (2020).
[25] J. de Rojas, J. Salguero, F. Ibrahim, M. Chshiev, A. Quintana, A. Lopeandia, M. O. Liedke, M. Butterling, E. Hirschmann, A. Wagner, L. Abad, J. L. Costa-Krämer, E. Menéndez, and J. Sort, Magneto-ionics in single-layer transition metal nitrides, ACS Appl. Mater. Interfaces 13, 30826 (2021).

[26] M. Kimura, S. Inoue, T. Shimoda, and T. Sameshima, Device simulation of grain boundaries in lightly doped polysilicon films and analysis of dependence on defect density, Jpn. J. Appl. Phys., Part 1 Regul. Pap. Short Notes Rev. Pap. 40, 49 (2001).

[27] R. Landauer, in AIP Conference Proceedings, Vol. 40 (AIP Publishing, 1978), pp. 2-45.

[28] C. Durkan and M. Welland, Size effects in the electrical resistivity of polycrystalline nanowires, Phys. Rev. B 61, 14215 (2000).

[29] R. Resta and S. Sorella, Electron Localization in the Insulating State, Phys. Rev. Lett. 82, 370 (1999).

[30] W. Kohn, Theory of the insulating state, Phys. Rev. 133, A171 (1964).

[31] ÁM Pendás, J. M. Guevara-Vela, D. M. Crespo, A. Costales, and E. Francisco, An unexpected bridge between chemical bonding indicators and electrical conductivity through the localization tensor, Phys. Chem. Chem. Phys. 19, 1790 (2017).

[32] J. Szczyrbowski and K. Schmalzbauer, DC conductivity in polycrystalline metals for bulk and thin-film samples, J. Phys. F Met. Phys. 16, 2079 (1986).

[33] A. F. Mayadas and M. Shatzkes, Electrical-resistivity model for polycrystalline films: The case of arbitrary reflection at external surfaces, Phys. Rev. B 1, 1382 (1970).

[34] L. Moraga, C. Arenas, R. Henriquez, S. Bravo, and B. Solis, The electrical conductivity of polycrystalline metallic films, Phys. B Condens. Matter 499, 17 (2016).

[35] T. T. Tsong, Field penetration and band bending near semiconductor surfaces in high electric fields, Surf. Sci. 81, 28 (1979).

[36] E. Stern, R. Wagner, F. J. Sigworth, R. Breaker, T. M. Fahmy, and M. A. Reed, Importance of the debye screening length on nanowire field effect transistor sensors, Nano Lett. 7, 3405 (2007).

[37] P. Debye and E. Hückel, Zur theorie Der elecktrolyte, Phys. Zeitschrift 24, 185 (1923).

[38] J. Mizsei, How can sensitive and selective semiconductor gas sensors Be made?, Sensors Actuators B. Chem. 23, 173 (1995).

[39] R. Gupta, N. Pandey, A. Tayal, and M. Gupta, Phase formation, thermal stability and magnetic moment of cobalt nitride thin films, AIP Adv. 5, 097131 (2015).

[40] K. H. Mader, F. Thieme, and A. Knappwost, Magnetische eigenschaften Der Kobalt-Stickstoff-phase $\mathrm{Co}_{3} \mathrm{~N}$, Zeitschrift Für Anorg. Und Allg. Chemie 366, 274 (1969).

[41] K. Suzuki, T. Kaneko, H. Yoshida, H. Morita, and H. Fujimori, Crystal structure and magnetic properties of the compound CoN, J. Alloys Compd. 224, 232 (1995).

[42] I. Krylov, E. Zoubenko, K. Weinfeld, Y. Kauffmann, X. $\mathrm{Xu}, \mathrm{D}$. Ritter, and M. Eizenberg, Obtaining low resistivity $(\sim 100 \mu \Omega \mathrm{Cm})$ TiN films by plasma enhanced atomic layer deposition using a metalorganic precursor, J. Vac. Sci. Technol. A 36, 051505 (2018). 
[43] D. S. Jeong, H. Schroeder, and R. Waser, Mechanism for bipolar switching in a $\mathrm{Pt} / \mathrm{TiO}_{2} / \mathrm{Pt}$ resistive switching cell, Phys. Rev. B 79, 195317 (2009).

[44] R. Waser, R. Dittmann, G. Staikov, and K. Szot, Redoxbased resistive switching memories - nanoionic mechanisms, prospects, and challenges, Adv. Mater. 21, 2632 (2009).

[45] A. Wagner, M. Butterling, M. O. Liedke, K. Potzger, and R. Krause-Rehberg, Positron annihilation lifetime and Doppler broadening spectroscopy at the ELBE facility, AIP Conf. Proc. 1970, 040003 (2018).

[46] E. Hirschmann, M. Butterling, U. Hernandez Acosta, M. O. Liedke, A. G. A. Elsherif, P. Petring, M. Görler, R. Krause-Rehberg, and A. Wagner, A new system for real-time data acquisition and pulse parameterization for digital positron annihilation lifetime spectrometers with high repetition rates, J. Instrum. 16, P08001 (2021).

[47] L. Lutterotti and P. Scardi, Simultaneous structure and size-strain refinement by the rietveld method, J. Appl. Crystallogr. 23, 246 (1990).

[48] A. Quintana, J. Zhang, E. Isarain-Chávez, E. Menéndez, R. Cuadrado, R. Robles, M. D. Baró, M. Guerrero, S. Pané, B. J. Nelson, C. M. Müller, P. Ordejón, J. Nogués, E. Pellicer, and J. Sort, Voltage-induced coercivity reduction in nanoporous alloy films: A boost toward energyefficient magnetic actuation, Adv. Funct. Mater. 27, 1 (2017).

[49] C. Navarro-Senent, A. Quintana, E. Menéndez, E. Pellicer, and J. Sort, Electrolyte-gated magnetoelectric actuation: Phenomenology, materials, mechanisms, and prospective applications, APL Mater. 7, 030701 (2019).

[50] K. K. Mahanta, G. C. Mishra, and M. L. Kansal, Estimation of electric double layer thickness from linearized and nonlinear solutions of poisson-boltzman equation for single type of ions, Appl. Clay Sci. 59-60, 1 (2012).

[51] L. Yang, B. H. Fishbine, A. Migliori, and L. R. Pratt, Dielectric saturation of liquid propylene carbonate in electrical energy storage applications, J. Chem. Phys. 132, 044701 (2010).

[52] H. Wang and L. Pilon, Accurate simulations of electric double layer capacitance of ultramicroelectrodes, J. Phys. Chem. C 115, 16711 (2011).

[53] M. Gong, J. Gao, D. Shen, P. Li, W. Tong, and C. Liu, Electromagnetic wave absorption properties in $\mathrm{Ku}$-band of magnetic iron nitrides prepared by high energy ball milling, J. Magn. Magn. Mater. 514, 167246 (2020).

[54] M. González and A. Ibarra, Dielectric behaviour of commercial polycrystalline aluminum nitride, Diam. Relat. Mater. 9, 467 (2000).

[55] See Supplemental Material at http://link.aps.org/supple mental/10.1103/PhysRevApplied.16.034042 which includes refs [71-74] for further magnetic, electronic, and magnetoelectric characterization, as well as computational modeling.

[56] S. H. Cho, K. R. Yoon, K. Shin, J. W. Jung, C. Kim, J. Y. Cheong, D. Y. Youn, S. W. Song, G. Henkelman, and I. D. Kim, Synergistic coupling of metallic cobalt nitride nanofibers and IrOx nanoparticle catalysts for stable oxygen evolution, Chem. Mater. 30, 5941 (2018).
[57] P. Chen, K. Xu, Y. Tong, X. Li, S. Tao, Z. Fang, W. Chu, X. $\mathrm{Wu}$, and $\mathrm{C}$. $\mathrm{Wu}$, Cobalt nitrides as a class of metallic electrocatalysts for the oxygen evolution reaction, Inorg. Chem. Front. 3, 236 (2016).

[58] J. V. Olsen, P. Kirkegaard, N. J. Pedersen, and M. Eldrup, PALSfit: A new program for the evaluation of positron lifetime spectra, Phys. Status Solidi Curr. Top. Solid State Phys. 4, 4004 (2007).

[59] R. Krause-Rehberg and H. S. Leipner, Positron Annihilation in Semiconductors, XV (1999).

[60] F. Tuomisto and I. Makkonen, Defect identification in semiconductors with positron annihilation: Experiment and theory, Rev. Mod. Phys. 85, 1583 (2013).

[61] K. Wada and T. Hyodo, A simple shape-free model for pore-size estimation with positron annihilation lifetime spectroscopy, J. Phys. Conf. Ser. 443, 012003 (2013).

[62] M. O. Liedke, W. Anwand, R. Bali, S. Cornelius, M. Butterling, T. T. Trinh, A. Wagner, S. Salamon, D. Walecki, A. Smekhova, H. Wende, and K. Potzger, Open volume defects and magnetic phase transition in Fe60Al40 transition metal aluminide, J. Appl. Phys. 117, 163908 (2015).

[63] T. Broom, Lattice defects and the electrical resistivity of metals, Nature 171, 1104 (1953).

[64] M. Weisheit, S. Fähler, A. Marty, Y. Souche, C. Poinsignon, and D. Givord, Electric field-induced modification of magnetism in thin-film ferromagnets, Science 315, 349 (2007).

[65] S. Robbennolt, E. Menéndez, A. Quintana, A. Gómez, S. Auffret, V. Baltz, E. Pellicer, and J. Sort, Reversible, electric-field induced magneto-ionic control of magnetism in mesoporous cobalt ferrite thin films, Sci. Rep. 9, 10804 (2019).

[66] S. Robbennolt, A. Nicolenco, P. Mercier Fernandez, S. Auffret, V. Baltz, E. Pellicer, E. Menéndez, and J. Sort, Electric field control of magnetism in iron oxide nanoporous thin films, ACS Appl. Mater. Interfaces 11, 37338 (2019).

[67] C. Leighton, Electrolyte-based ionic control of functional oxides, Nat. Mater. 18, 13 (2019).

[68] B. Balasubramanian, X. Zhao, S. R. Valloppilly, S. Beniwal, R. Skomski, A. Sarella, Y. Jin, X. Li, X. Xu, H. Cao, H. Wang, A. Enders, C. Z. Wang, K. M. Ho, and D. J. Sellmyer, Magnetism of new metastable cobalt-nitride compounds, Nanoscale 10, 13011 (2018).

[69] J. R. Rumble, editor, CRC Handbook of Chemistry and Physics, 97th ed. (CRC Press, Boca Raton, FL, USA, 2016).

[70] J. Häglund, A. Fernández Guillermet, G. Grimvall, and M. Körling, Theory of bonding in transition-metal carbides and nitrides, Phys. Rev. B 48, 11685 (1993).

[71] M. J. Puska and R. M. Nieminen, Defect spectroscopy with positrons: A general calculational method, J. Phys. F Met. Phys. 13, 333 (1983).

[72] A. P. Seitsonen, M. J. Puska, and R. M. Nieminen, Realspace electronic-structure calculations: Combination of the finite-difference and conjugate-gradient methods, Phys. Rev. B 51, 14057 (1995).

[73] D. L. Leslie-Pelecky and R. D. Rieke, Magnetic properties of nanostructured materials, Chem. Mater. 8, 1770 (1996).

[74] W. Anwand, G. Brauer, M. Butterling, H. R. Kissener, and A. Wagner, Design and construction of a slow positron beam for solid and surface investigations, Defect Diffus. Forum 331, 25 (2012). 